\title{
Are 2 procedures really better than 1 for patients undergoing coronary artery bypass graft procedure?
}

\author{
Michel Carrier, MD, and Yoan Lamarche, MD
}

From the Department of Surgery, Montreal Heart Institute, University of Montreal, Montreal, Quebec, Canada. Disclosures: Authors have nothing to disclose with regard to commercial support.

Received for publication April 30, 2018; revisions received April 30, 2018; accepted for publication May 2, 2018; available ahead of print June 22, 2018.

Address for reprints: Michel Carrier, MD, Department of Surgery, Montreal Heart Institute, 5000 Belanger St E, Montreal, Quebec, Canada H1T1C8 (E-mail: michel.carrier@icm-mhi.org).

J Thorac Cardiovasc Surg 2018;156:1798

$0022-5223 / \$ 36.00$

Copyright (c) 2018 by The American Association for Thoracic Surgery

https://doi.org/10.1016/j.jtcvs.2018.05.018

Patel and colleagues ${ }^{1}$ report the results of hybrid coronary revascularization (HCR) procedure consisting of left internal thoracic artery (LITA) harvest facilitated with a robotic system, off-pump LITA to left anterior descending artery (LAD) anastomosis through an anterior minithoracotomy, and in most cases a second subsequent percutaneous coronary artery intervention procedure with stents deployed in the circumflex or right coronary artery in patients with double-vessel disease (including left main stenosis). Two hundred seven patients were compared with a group of patients who underwent traditionally performed coronary artery bypass grafting (CABG) surgery defined as LITA to LAD anastomosis plus saphenous vein graft, in most cases performed through standard sternotomy and off-pump techniques. Baseline characteristics were similar in both groups in a propensity-matched analysis.

Hospital mortality and 8-year patient survival was similar in both groups despite 2 patients in the HCR group who required immediate reoperation for LITA-LAD occlusion and 3 patients who underwent the traditional $\mathrm{CABG}$ approach where a saphenous vein graft was used to perform the LAD anastomosis. A greater number of patients undergoing traditional $\mathrm{CABG}$ procedure required blood transfusion and the patients undergoing HCR enjoyed shorter hospital stays.

Although the authors concluded that HCR has similar clinical results and utilizes fewer perioperative resources, we should take a closer look at the data. Patients of both groups were young (average age, 65 years), one-third were women, and half had diabetes with a normal ejection fraction. Forty-eight of 207 patients (23\%) required $89 \mathrm{mi}$ nutes of on-pump time and 63 minutes of aortic crossclamp time to complete the 2 coronary anastomoses in the traditional group, which is longer than expected. Moreover, women had a much higher risk of mortality throughout the study period (hazard ratio, 2.47; 95\% confidence interval, 1.02-5.6) within the HCR group.

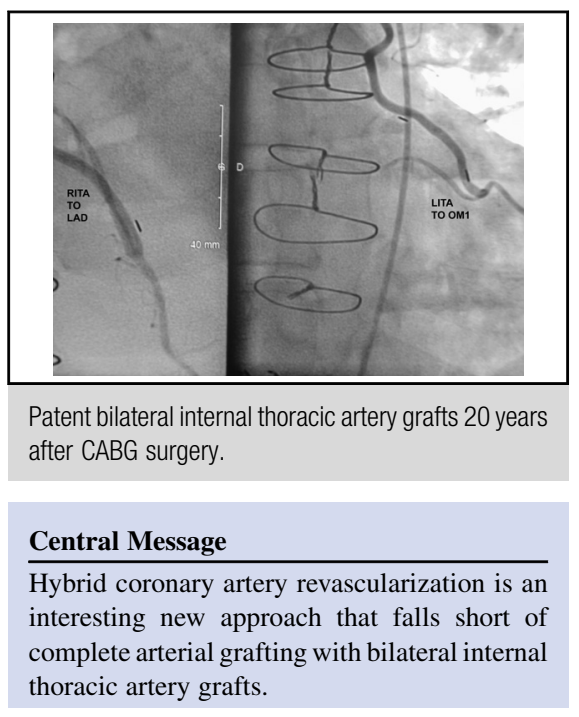

See Article page 1799 .

It is obvious that the proposed hybrid approach offers several advantages to patients; namely, a minimally invasive technique with shorter surgical incisions, less thoracic trauma with potentially less pain, fewer blood transfusions, and shorter hospital stay with similar medium-term survival. It also appears that these benefits come with significant costs, the use of saphenous vein graft instead of arterial grafts, higher risk of mortality in women, the additional risk of 2 procedures instead of 1, and the long-term need for dual antiplatelet therapy in an early postoperative setting.

We have 3 main objections to this approach. First, most patients could have benefited from complete arterial revascularization, including double ITA grafts. ${ }^{2}$ Second, women are at higher risk of death within the HCR group. Third, the addition of procedure-related risks should be justified based on hard evidence of benefits, not on similar medium-term survival. Otherwise, 1 intervention during a single hospital stay is better than 2 for patients undergoing $\mathrm{CABG}$.

\section{References}

1. Patel NC, Hemli JM, Kim MC, Seetharam K, Pirelli L, Brinster DR, et al. Shortand intermediate-term outcomes of hybrid coronary revascularization for double vessel disease. J Thorac Cardiovasc Surg. 2018;156:1799-807.

2. Aldea GS, Bakaeen FG, Pal J, Fremes S, Head SJ, Sabik J, et al. The Society of Thoracic Surgeons clinical practice guideline on arterial conduits for coronary artery bypass grafting. Ann Thorac Surg. 2016;101:801-9. 